\title{
A Hierarchical multi-classifier Framework for Landform Segmentation using Multi-spectral Satellite Images - A case study over the Indian subcontinent
}

\author{
Utthara Gosa Mangai, Suranjana Samanta, Sukhendu Das \\ VP Lab, Dept. of CSE \\ IIT Madras, Chennai, India \\ utthara@cse.iitm.ac.in,ssamanta@cse.iitm.ac.in,sdas@iitm.ac.in
}

\author{
Pinaki Roy Chowdhury \\ Defence Terrain Research Laboratory (DRDO) \\ Delhi, India \\ rcpinaki@yahoo.com
}

\author{
Koshy Varghese \\ Department of Civil Engineering \\ IIT Madras, Chennai, India \\ koshy@iitm.ac.in
}

\author{
Manisha Kalra \\ Parc Vista Condo, 460 Corporation Road, \#03-08 \\ Singapore, 649815 \\ manishakalra007@gmail.com
}

\begin{abstract}
There is an increasing need for automatically segmenting the regions of different landforms from a multispectral satellite image. The problem of Landform classification using data only from a 3-band optical sensor (IRS-series), in the absence of DEM (Digital Elevation Model) data, is complex due to overlapping and confusing spectral reflectance from several different landform classes. We propose a hierarchical method for landform classification for identifying a wide variety of landforms occurring over parts of the Indian subcontinent. At the first stage, the image is classified into one of three broad categories: Desertic, Coastal or Fluvial, using decision fusion of three SVMs (Support Vector Machine). In the second stage, the image is then segmented into different regions of landforms, specifically belonging to the class (category) identified at stage 1. To show the improvement in accuracy of our classification method, the results are compared with two other methods of classification.
\end{abstract}

Keywords-Landform Classification; Support Vector Machine; Hierarchical Classification; Decision Fusion;

\section{INTRODUCTION}

Landform classification is a problem of identifying a set of predefined categories of landforms. Probabilistic classification of landforms is significant and a hard problem because of the versatility and variations in the categories of landforms, their patterns, features, association rules and signatures. However, there exists huge scope of application of such an area of work, in remote sensing and GIS (Geographic Information System), trafficability and surveillance in military domain, cartographical updates and geological surveys etc. We pose the problem as: Given a satellite image of a large area, identify the different landform segments from the image, with a confidence measure for each detected landform segments.

Indian Landform image data and the corresponding handlabeled data as ground truth were provided to us by GIS experts. Landform classification had been attempted in the past either using data from SAR (Synthetic Aperture Radar) or DEM or by integrating data form multiple sensors. The objective of our research was to classify the Indian landforms from a single sensor (3-band) image. This objective led to the development of a hierarchical classification scheme. Since different landforms have different signatures, a single classifier will not be sufficient for classification. Hence, a hierarchical landform classification method is proposed, where separate sets of training samples are used for separate classifiers at different levels of the hierarchical framework.

Related works have been done by [1], [2], [3], [4], [5], [6] to solve the problem mostly using DEM data. The methods used for landform classification can be broadly divided into three different categories: (i) Use of a statistical (Bayes, Fuzzy k-means, Maximum Likelihood etc.) criteria; (ii) Set of rules in a framework for multi-level thresholding (with different parameters); and (iii) Neural classifierSelf-organising map (SOM), feed forward neural network (FFNN) etc. In all of these cases, results have not been shown in the absence of DEM, which seems to provide a strong clue for visual experts to detect and discriminate closely lying neighboring landforms. The main problem with the use of DEM is its free availability at a higher resolution (below 10 or $25 \mathrm{~m}$, say) and online accessibility for a particular region based on instantaneous demand.

In [3], a numerical method for classification and characterization of landforms on Mars was proposed. Topographic attributes were calculated from DEM and classified using SOM. Landform of 21 classes were broadly classified into five categories (Highlands, craters, lowlands, high-relief and channels). Reference [7] reports results of employing a fuzzy c-means classification for a DEM data. Remotely sensed topographic data gathered by orbiting satellites were transformed into semantically meaningful maps of landforms [8]. The mapping is achieved by means of scene seg- 
mentation using K-means-based agglomerative segmentation and watershed-based segmentation followed by supervised classification of segments. Hosokawat and Hoshi [1] used SOM to classify landforms into hill, plateau, fan based on a land cover map and DEM and reported an accuracy of $77 \%$ using four landform samples. Reference [4] used data from multiple sensors and proposed an approach which employs a class dependent feature selection in conjunction with pairwise Bayesian classifiers and reports an accuracy of $96 \%$. Hengl and Rossiter [5] used maximum-likelihood classifier to classify landforms into 21 legends. Authors used DEM data to extract nine terrain parameters. An accuracy of $97 \%$ was also reported by iterative selection of point sample training set.

In spite of the vastness of the work done on landform classification, it can be seen that all of these require either DEM (in most cases) or SAR/ LIDAR data. For certain online applications the DEM data may not be available (as it is not a direct output of a sensor). It remains a challenging task and a hard problem to perform landform classification using an optical sensor image alone as input. This is the main reason why researchers have not been done to solve this problem with a reasonable degree of accuracy using only a multi-band optical image. These has been prime motivation of our work. We designed a hierarchical multi-classifier framework to solve this problem. Section II describes the overall framework and various stages in Hierarchical Landform Classification. Results are discussed in Section III. Section IV presents the conclusion of the paper.

\section{PROPOSED FRAMEWORK}

Based on interactions with GIS and Geomorphological experts, we came to understand that the data samples were acquired from three (3) major categories of areas/zones: Desertic, Coastal and Fluvial. We hence used a divide and conquer approach to design a hierarchical system of classification. This made the problem tractable, as about 30 different classes of landforms were available for segmentation, and the features (spectral) were overlapping and confusing over many classes of landforms. In the following, we first discuss the three major categories of landform classes followed by the design of our proposed framework.

1) Fluvial landforms: These consist of the landforms that are produced by the action of stream or river. The following landforms classes are being considered for the purpose of landform identification from satellite images of fluvial areas: (a) Active - Active Channel, Bars, Flood Plains, Alluvial Plains, (b) Relict (Dry) -Oxbow lakes, Forested swamps, Alluvial Plains.

2) Coastal landforms: The following landform classes are being considered for the purpose of landform identification from satellite images of the coastal belt:
Creek, Forested Swamp, Sandy Beach, Coastal Bars, Sea, Flood Plains and Alluvial Plains.

3) Desertic landforms: The following classes of landforms have been considered for detection from satellite images taken from desert areas: Barchan Dunes, Longitudinal Dunes, Transverse Dunes, Parabolic Dunes, Barchanoid, Sandy Plains, Rocky Exposures, Inselberg, Remnant Stony Surfaces (Salt Flats) and Playa.

The proposed hierarchical classification scheme is shown in Fig. 1. We assume that an input image given to the landform extraction system will not contain different types of landforms belonging to any two of the three different categories: Desertic, Coastal and Fluvial (termed as the Super-Group classes). We propose a hierarchical method of landform classification (Fig. 1) that performs super-group classification at the first level i.e. it determines the supergroups of the input image. The uncertainty of classification into different landform zones is the largest at this stage and a near $100 \%$ crisp classification is expected from the supergroup classifier in use. This enables us to search for the probable set of landforms occurring in the input image, only under the particular super-group that has been determined at the first step of processing. Thus, the uncertainty involved in detecting the landforms in an image, is reduced to approximately one-third of the total number of classes. The $2^{n d}$ stage of processing detects sub-classes of a landform category (i.e. either Desertic or Coastal or Fluvial) detected at the super-group stage.

At the leaves of the hierarchical classification tree given in Fig. 1, are all the landforms of interest organized suitably under their respective processing methodologies. Based on the output of the super-group classifier, all algorithms tailored for the extraction of features of each subset of landforms would be used in parallel for accurate segmentation of leaf landform nodes. Thus, at the bottommost level of the hierarchical tree, landform-specific modules are used to detect landforms accurately. Such modularity in the nature of landform extraction is designed, keeping in mind the huge amount of uncertainty, combinations of occurrences and adjacency of various landforms as well as the subjectivity in the landform definitions, which are undoubtedly too confusing for any one-classifier to capture. The nodes used to label the different processing methods, as given in Fig. 1 are: DP-Desertic Processing, CP-Coastal Processing and FP-Fluvial Processing methods. These processing methods are discussed in detail in the following.

\section{A. Super-Group Classification}

This is the topmost stage of the proposed hierarchical classification as shown in Fig. 1. A Support Vector Machine (SVM) based classification technique has been adopted in our design for the task of identifying an input image as belonging to one of the Desertic, Coastal or Fluvial landform Super-Group categories. In order to capture and exploit 


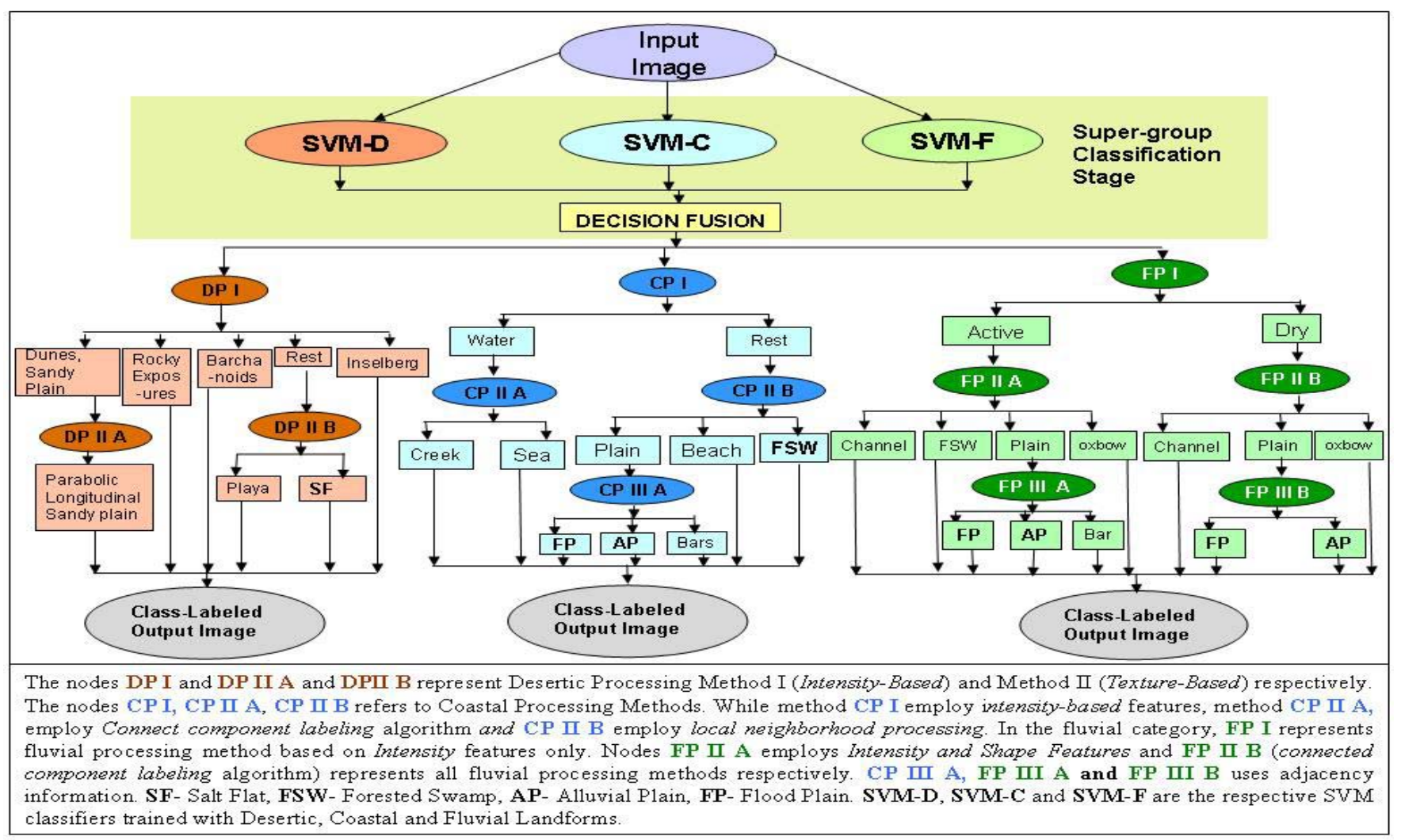

Figure 1: The proposed hierarchical classification scheme.

the variability among the different multi-spectral images belonging to each of the super-groups, histograms of all the 3 bands, namely NIR, Red and Green were used as features for classification. Thus the classification at this stage is primarily based on the multi-spectral intensities (color) features, using SVM as a classifier. The number of samples used for training and testing, and the accuracies of classification obtained are given in Table I. Sum rule [9] has been used for fusing the decisions, as it has been shown to work better in [10].

Table II shows the number of testing samples misclassified during testing phase before fusion, by each of the three classifiers (SVM-D, SVM-C and SVM-F). SVM-D denotes the SVM classifier trained with 150 samples of Desertic landforms and 300 from the remaining two classes. In a similar way, SVM-F and SVM-C were also trained. All the SVM classifiers are used with a polynomial kernel (of degree 2). Number of misclassified samples in table II of each classifier includes False Rejection and False acceptance. Accuracy was improved by $1.3 \%$ (average) after using decision fusion technique. Details of the misclassified samples and the overall accuracy at the super-group stage of classification, before and after fusion are given below:

Number of misclassified samples before Fusion: 16
Table I: Accuracy Obtained During Testing Phase For Different Landforms.

\begin{tabular}{|c|c|c|c|c|}
\hline \multirow{2}{*}{ Landforms } & \multicolumn{2}{|c|}{ Samples } & \multicolumn{2}{c|}{ Accuracy in \% } \\
\cline { 2 - 5 } & Training & Testing & Before fusion & After fusion \\
\hline Desertic & 150 & 250 & 99.07 & 99.47 \\
\hline Fluvial & 150 & 250 & 99.47 & 100 \\
\hline Coastal & 150 & 250 & 99.2 & 99.6 \\
\hline
\end{tabular}

Table II: Different Classifiers And Their Misclassified Samples During Testing Before Fusion.

\begin{tabular}{|l|c|c|c|}
\hline \multirow{2}{*}{ Classifier } & \multicolumn{2}{|c|}{ Samples } & Misclassified Samples \\
& braining & Testing & before fusion \\
\hline SVM-D & 450 & 750 & 7 \\
\hline SVM-F & 450 & 750 & 4 \\
\hline SVM-C & 450 & 750 & 6 \\
\hline
\end{tabular}

Number of misclassified samples after Fusion: 7 Overall Accuracy before fusion: $\quad 97.87 \%$ Overall Accuracy after fusion: $\quad 99.07 \%$. 


\section{B. Sub-Group Classification}

This stage consists of a set of processes which are detailed at the bottom part of the flowchart in Fig. 1, following super-group classification. Based on the experimentations with landform samples, as well as discussions with geoscientists interacting with us, it was observed that we need to design a hierarchical framework tailored for the extraction of a particular landform of interest (being analyzed), because different landforms may require different methods of processing. In the following, we discuss the processing methodologies used to obtain results on the landform data samples supplied to us by experts. The four main features used for classification are:

- Local mean and/or variance of the approximation subband of the DWT (Discrete Wavelet Transform) for all the three bands (NIR, R and G). Daubeschies 10-tap filter is used to extract the DWT coefficients which represent the texture features in our case.

- Local mean and/or variances of the multi-resolution color intensities of three bands (NIR, R and G).

- Spatial adjacency of the landforms are stored in the form of an adjacency matrix, which was formulated using domain knowledge obtained from GIS experts.

- Connected Component Labeling is used to extract the shape features which are used to classify certain shapes.

In the following three sub-sections, we present the properties, features and methods used to analyze three major categories of landforms discussed in this work.

1) Processing Modules for the Desertic Type of Landforms: Two types of processing occur predominantly for extraction of desertic landforms. These can thus be grouped together as DP-I and DP-II, each corresponding to methods based on texture features and shape features, respectively. The steps of processing for identification of landform in desertic images are as follows:

- DP-I (multi-class SVM) uses a SVM trained using local mean from 'Approximation' of DWT of all three spectral bands (NIR, R and G), for differentiating between Dunes, Salt flats, Rocky exposure, Barchanoids and Inselberg.

- DP-II A (Template Matching), further classifies dunes into parabolic, longitudinal or sandy plain. Templates for parabolic and longitudinal dunes are obtained from the training samples. The templates are matched with the landform image using cross correlation. Output of this operation is compared with two thresholds (one for parabolic and another for longitudinal) for labeling as parabolic or longitudinal dunes. The rest are labeled as Sandy Plains.

- DP-II B (Area-based operation): Both saltflats and Playa have similar signatures, it was observed that Playa was comparatively smaller than salt flats.
2) Processing Modules for the Coastal Type of Landforms: It can be observed that intensity-based features have a major role to play for extraction of coastal landforms. This is possibly because of the small resolution, fineness and non-texture information that most of the coastal landforms have been found to possess. Association rules have also been employed in order to encode domain-experts knowledge in observing certain key characteristics of coastal landforms within the system. The steps of processing for identification of landform in coastal images are as follows:

- CP-I (Threshold) segregates the water-bodies from land by thresholding the intensity of blue color (in NIR, R and $\mathrm{G}$ band). If the intensity is very less it is considered as water-bodies otherwise it is considered as land.

- CP-II A (Adjacency Information) uses adjacency information (nearness using an Euclidean measure) to identify creeks from the water bodies. Creeks are detected by identifying fine and narrow extents of water bodies extending into the land. This is done by observing within a small window whether the extent of sea is covered by land on the both the sides. This decision is taken by observing whether the pixels and the border of the window are non-sea type. The rest of the water bodies are detected as sea.

- CP-II B (SVM) classifies plain, beach and forested swamp using a SVM trained using the mean of multiresolution color intensity features, computed as:

$$
X_{i, j}=\left[\mu\left(I_{i, j}^{n}\right) \mu\left(I_{i, j}^{r}\right) \mu\left(I_{i, j}^{g}\right)\right]
$$

where, $X_{i, j}$ represents a $3 \mathrm{D}$ feature vector corresponding to $(i, j)^{t h}$ pixel. $I_{i, j}^{n}, I_{i, j}^{r}$ and $I_{i, j}^{g}$ represents intensity values of $(i, j)^{t h}$ pixel in three spectral bands (NIR, R and G) of the input image respectively and $\mu(h)$ represents the mean of $h$ computed using windows of size $5 * 5,17 * 17$ and $31 * 31$.

- CP-III A (Connected Component Labeling \& Adjacency Information)

- Coastal bars possess unique characteristic property of being enclosed by sea on all sides. A connected component labeling algorithm [11] is employed over pixels whichever is classified as Plains (detected in CP II B) to determine if all set of connected pixels are surrounded by sea.

- Among different Plains (detected in CP II B) whichever is closer to creek, are classified as flood plains and others are classified as alluvial plain based on adjacency information.

3) Processing Modules for the Fluvial Type of Landforms: All the methods that have been employed for detection of fluvial landforms rely heavily on intensity-based features. Since fluvial landforms are produced by the action of river or an active channel, a satellite image taken of a fluvial area must therefore necessarily contain an active channel within 
it. The steps of processing for identification of landforms in fluvial images are as follows:

- FP -I (SVM) uses a multi-class SVM trained using histogram features of all three spectral bands, is used to differentiate between active and dry zones. The channels which were active were blue in color and others were red in color (based on NIR, R and G bands). Since the two signatures are different they are trained and classified separately.

- FP-II A and FP II B (SVM) uses a SVM classifier trained using mean of color features similar to CP II B. FP II A classifies channel, forested swamp, plain and ox-bow. FP II B classifies channel, plain and ox-bow.

- FP-III A (Connected Component Labeling \& Adjacency Information) identifies Bars, Flood Plain, and Alluvial Plain. FP III B (Adjacency Information) classifies alluvial Plain and flood plain. To identify Bars connected component-labeling algorithm [11] is used. Adjacency Information is used to categorize Plains into Flood Plain or Alluvial Plain. If a plain is near (in Euclidean sense) to active channels, it is considered to be 'Flood Plain', else if it does not have a common boundary with active channel, it is considered to be 'Alluvial Plain'.

- Bars possess a unique characteristic property of being enclosed by active channel on all sides. A connected component-labeling algorithm is employed to determine if all set of connected pixels whichever is classified as Plains (detected in FP II A) are enclosed by an active channel.

- Among different Plains detected (in FP II A and FP II B) whichever is close to active channel are classified as flood plains and others are classified as alluvial plain.

\section{RESULTS AND DISCUSSION}

Training and testing samples from the landform data were acquired with the help of hand-labeled data to improve the results of sub-classification stages. Results will be shown using the same and compared with prior work done by [12], [13]. The two stages of the landform classification scheme, as proposed in Section II, have been implemented. The first stage of super-group characterization consists of a fusion of three SVM classifiers. The second stage is a hierarchical organization, where the leaf nodes of the tree indicate the output for a particular landform, while the intermediate nodes consist of tailor-made processes, including SVM, connected component labeling, shape feature detection and labeling, etc.

Figs. 2, $3 \& 4$ shows the results obtained for landform images of different categories: Desertic, Coastal and Fluvial, where within each figure (a) is the Input image, (b) the corresponding hand-labeled data, (c) the output obtained by the unsupervised method [12], (d) the output obtained from the method proposed in [13] and (e) Output obtained from our proposed method. Each row in Figs. 2-4 use a unique color code (label) for each segment detected in the output map. Unsupervised method works well in case of landforms covering larger areas and are distinct like salt flats, Rocky exposure, sea etc. It fails in cases of dunes, ox-bow and Plains, where our proposed method gives better classification results. Almost all the landforms have been correctly identified by our algorithm, since different features were detected with suitable processing modules and suitable classifiers were used for identifying the different landform signatures. For example, template matching algorithm was used for identifying parabolic dunes and longitudinal dunes and connected component labeling algorithm was used for identifying creek and oxbow.

Table III shows the overall accuracy of classification for each landform obtained at sub-group level. In Table III, the classification accuracy has been shown for only those classifiers, for which we were able to compute this measure using a set of test samples. In other cases this was not estimated, as we had used trivial image processing methods (Template Matching, Connected Component Labeling, Adjacency Information, etc.) and not classifiers for segmentation and labeling of the pixels. The overall accuracy of the proposed method (quantitatively measured for a few landforms and visually compared in other cases using results on raster images) is better due to the hierarchical organization of a set of classifiers. Each classifier in our proposed framework, solves a specific part of the overall problem of classification, for a small set (2-4) of classes within a limited domain. Hence the performance of each classifier is quite high compared to the case of using a single classifier to solve the complete problem. A large number of classes having confusing or overlapping feature properties between classes and large dimension of the feature space would have made that single classifier provide unsatisfactory performance. Table IV shows the classification timing taken by different algorithms for different image sizes. Our proposed method takes lesser time than the unsupervised method [12], notably for larger size images. Though our method takes more time than the method proposed by Gagrani et al. [13], the performance of our method is superior (see Fig. 2-4).

\section{CONCLUSiOn AND Future WORK}

Hand-labeled data was obtained from GIS experts to compare the output produced by our algorithm. The proposed framework has been developed and tested successfully on all the samples given to us by GIS experts and the implementation (Matlab and Visual C++ environments) works up to an image size of $3000 \times 3000$. Fusion of DEM (high resolution) will definitely produce better results, but we intended to explore this complex problem in the absence of DEM. This approach gains significance because very accurate and high-resolution DEMs of unknown areas (especially new 
a
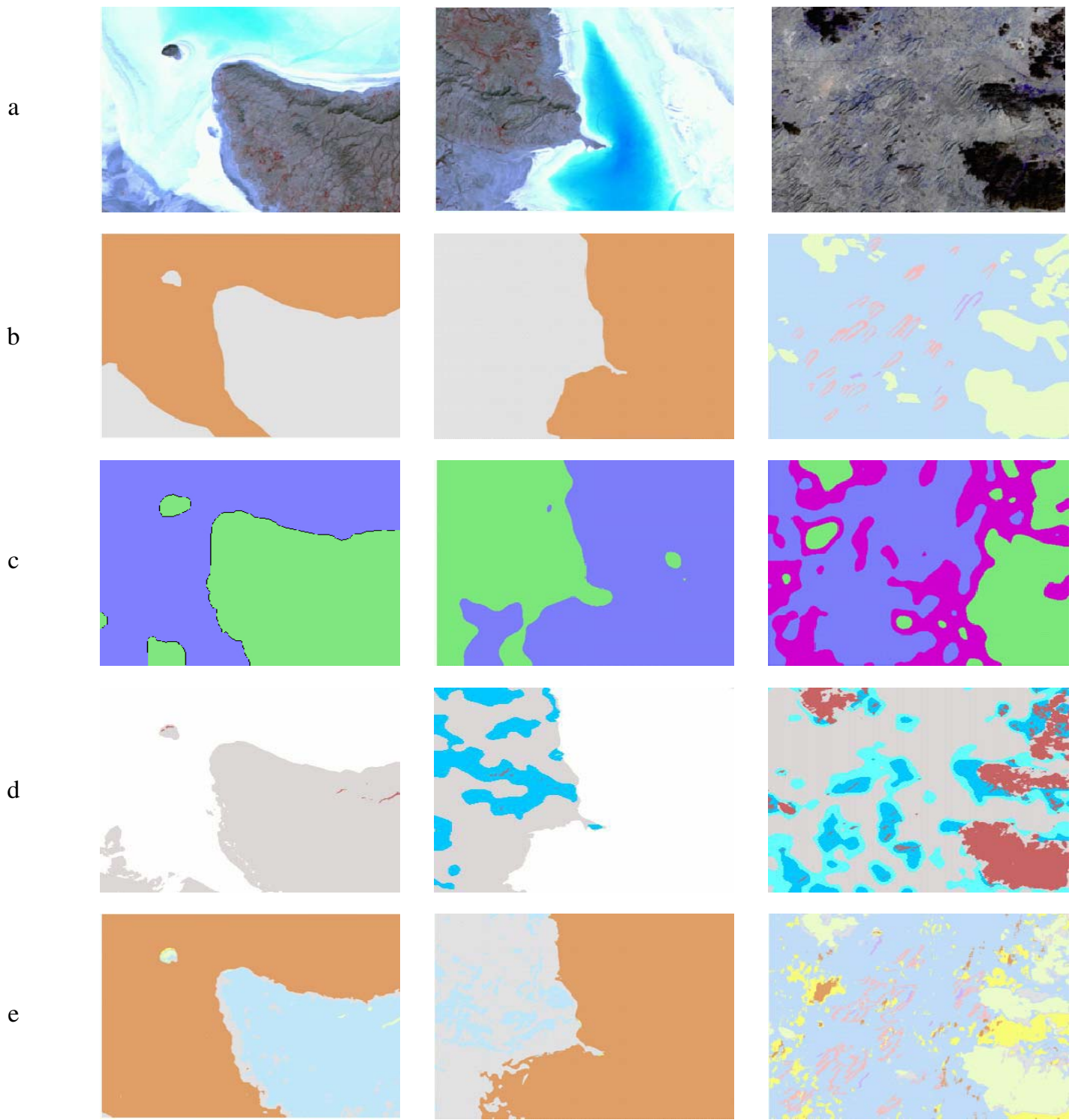

Figure 2: (a) Input Desertic image; b) Hand-Labeled Data; Output: (c) Unsupervised method [12] \& (d) Method proposed in [13]; (e) Our proposed method.

planets) and deserts are difficult to produce and are often not available for certain online applications (over areas of the Indian subcontinent).

\section{REFERENCES}

[1] M. Hosokawat and T. Hoshi, "Landform classification method using self-organizing map and its application to earthquake damage evaluation," in IEEE Geoscience and Remote Sensing Symposium, 2001, pp. 1684-86.

[2] H. Saadat, R. Bonnell, F. Sharifi, G. Mehuys, M. Namdar, and S. Ale-Ebrahim, "Landform classification from a digital elevation model and satellite imagery," Geomorphology, vol. 100, pp. 453-464, August 2008.
Table III: Performance Measures At Sub-group Classification Stage (See Fig. 1), For Only The Modules Involving Classifiers.

\begin{tabular}{|l|c|c|c|}
\hline \multirow{2}{*}{ Classifiers } & \multicolumn{2}{|c|}{ Samples } & Overall Accuracy \\
\cline { 2 - 3 } & Training & Testing & (in \%) \\
\hline DP I & 400 & 800 & 95.1 \\
\hline CP II B & 500 & 1200 & 96.56 \\
\hline FP II A & 300 & 600 & 94.09 \\
\hline FP II B & 200 & 600 & 96.64 \\
\hline
\end{tabular}

[3] B. D. Bue and T. F. Stepinski, "Automated classification of landforms on mars," Computers and Geosciences, vol. 32, 

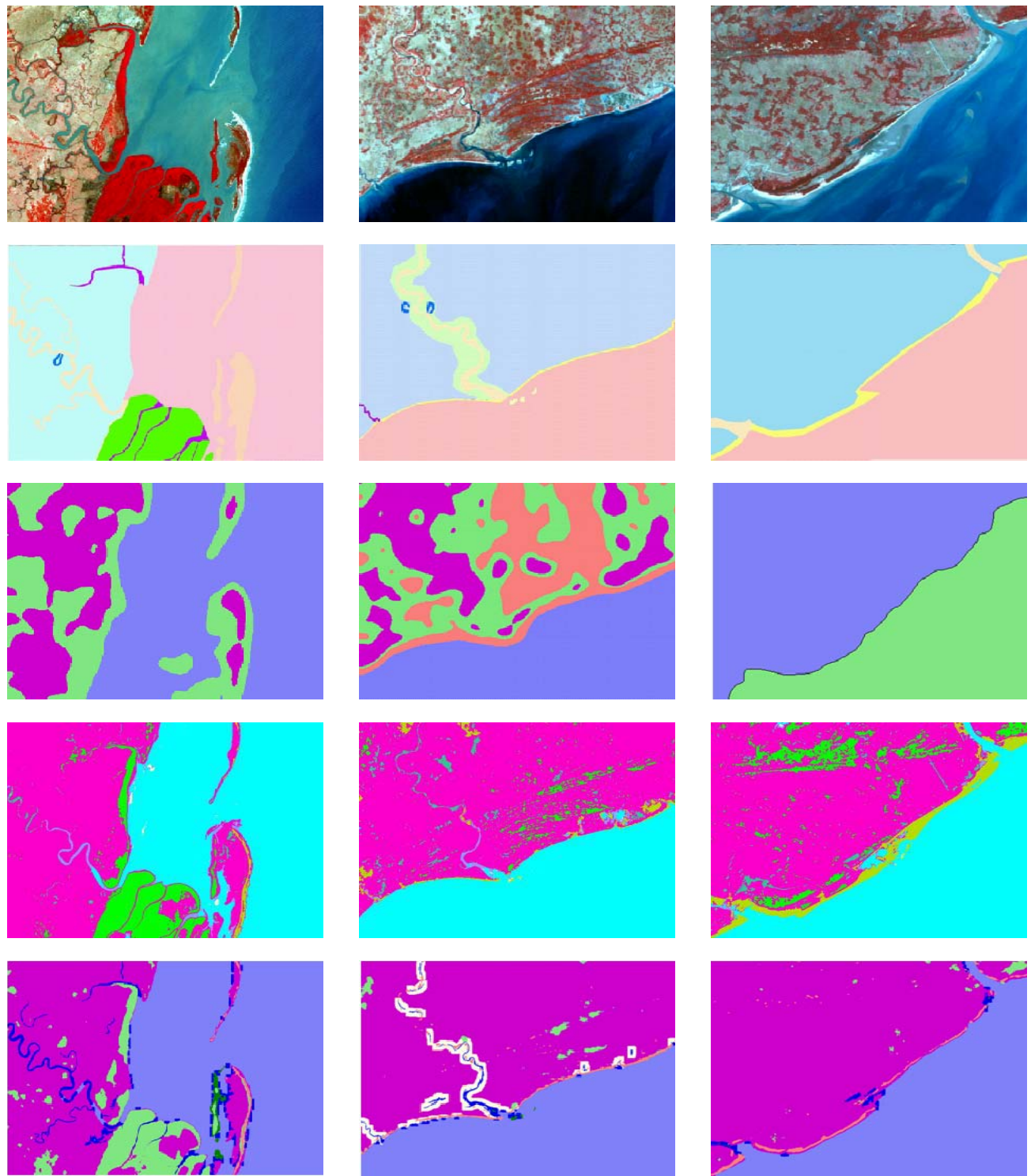

Figure 3: (a) Input Coastal image; b) Hand-Labeled Data; Output: (c) Unsupervised method [12] \& (d) Method proposed in [13]; (e) Our proposed method.

Table IV: Comparison Of The Computational Times (In Secs) For Classification, Taken By Different Methods.

\begin{tabular}{|c|c|c|c|}
\hline Image Size & $\begin{array}{l}\text { Unsupervised } \\
\text { Method [12] }\end{array}$ & $\begin{array}{l}\text { Method proposed } \\
\text { in [13] }\end{array}$ & Our Method \\
\hline $300 * 300$ & 34.81 & 31.56 & 32.42 \\
\hline $600 * 600$ & 772.19 & 98.97 & 112.54 \\
\hline $1000 * 1000$ & 14328.33 & 174.29 & 306.38 \\
\hline
\end{tabular}

no. 5, pp. 604-614, 2006.

[4] M. M. Crawford, S. Kumar, M. R. Ricard, J. C. Gibeaut, and A. Neuenschwander, "Fusion of airborne polarimetric and interferometric sae for classification of coastal environments," IEEE Transactions on Geoscience and Remote Sensing, vol. 37, no. 3, pp. 1306-15, May 1999.

[5] T. Hengl and D. G. Rossiter, "Supervised landform classification to enhance and replace photo-interpretation in semidetailed soil survey," Soil Science Society of America Journal, vol. 67 , no. 3, pp. 1810-22, 2003.

[6] P. A. Burrough, P. F. M. van Gaans, and R. A. MacMillan, "High-resolution landform classification using fuzzy kmeans," Fuzzy Sets Systems, vol. 113, no. 1, pp. 37-52, 2000.

[7] K. E. Arrell, P. F. Fisher, N. J. Tate, and L. Bastin, "A fuzzy c-means classification of elevation derivatives to extract the morphometric classification of landforms in snowdonia, 

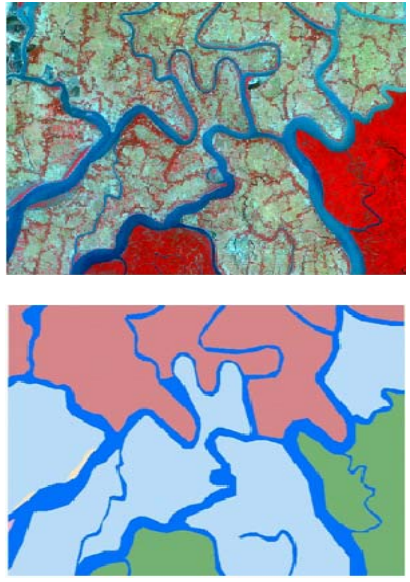

b

c
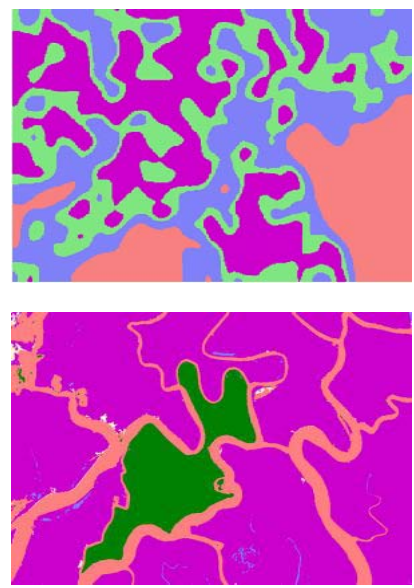

$\mathrm{d}$

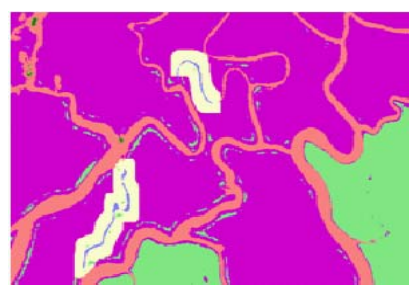

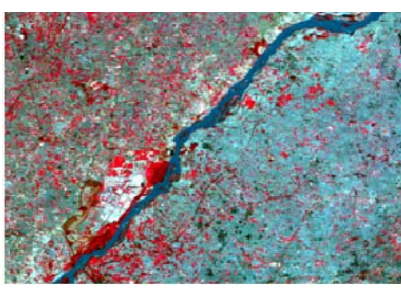
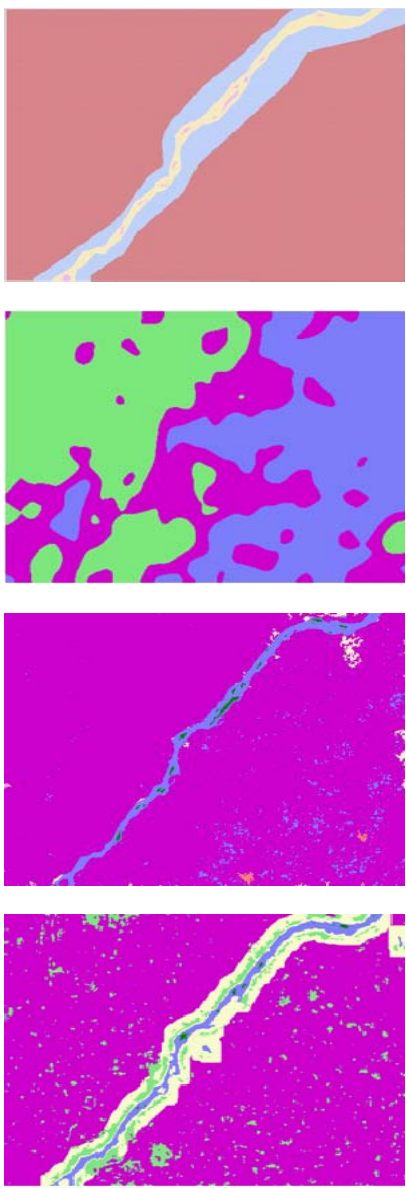
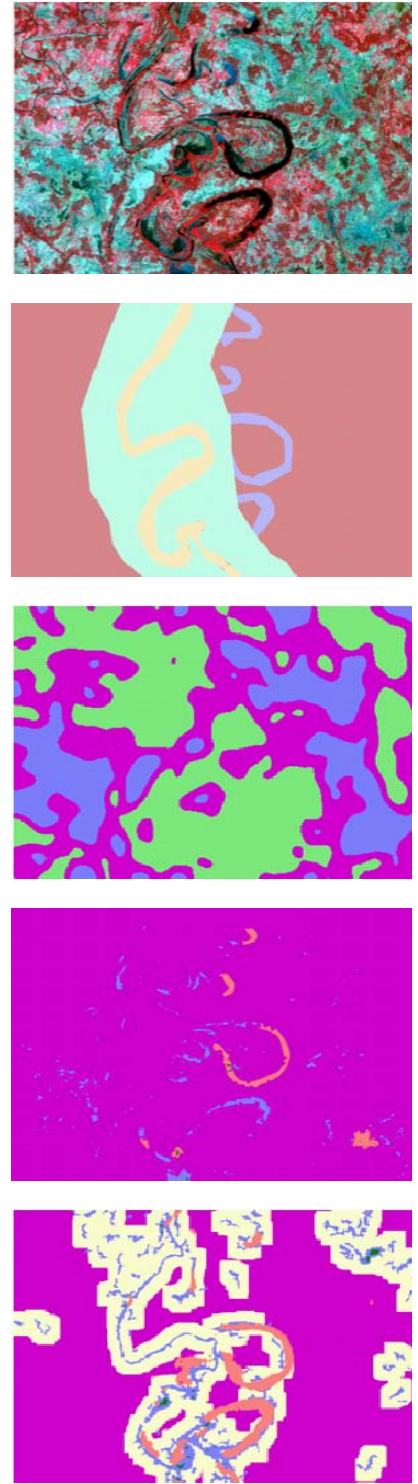

Figure 4: (a) Input Fluvial image; b) Hand-Labeled Data; Output: (c) Unsupervised method [12] \& (d) Method proposed in [13]; (e) Our proposed method.

wales," Computers and Geosciences, vol. 33, no. 10, pp. 1366-81, 2007.

[8] S. Ghosh, T. F. Stepinski, and R. Vilalta, "Automatic annotation of planetary surfaces with geomorphic labels," IEEE Transactions on Geoscience and Remote Sensing, vol. 48, no. 1 , pp. 175-185, 2010.

[9] L. Lam and C. Y. Suen, "Optimal combinations of pattern classifiers," Pattern Recognition Letters, vol. 16, pp. 945-954, September 1995.

[10] J. Kittler, M. Hatef, R. P. W. Duin, and J. Matas, "On combining classifiers," IEEE Transactions on Pattern Analysis and Machine Intelligence, vol. 20, no. 3, pp. 226-239, 1998.
[11] A. S. Glassner, "Fill 'er up!" IEEE Computer Graphics and Applications, vol. 21, no. 1, pp. 78-85, 2001.

[12] S. G. Rao, M. Puri, and S. Das, "Unsupervised segmentation of texture images using a combination of Gabor and wavelet features," in Indian Conference on Computer Vision, Graphics and Image Processing, 2004, pp. 370-375.

[13] A. Gagrani, L. Gupta, B. Ravindran, S. Das, P. Roychowdhury, and V. K. Panchal, "A hierarchical approach to landform classification of satellite images using a fusion strategy," in Indian Conference on Computer Vision, Graphics and Image Processing (Lecture Notes in Computer Science, 4338), 2006, pp. $140-151$. 